\title{
Diagnostic Value of Serum D-Dimer for Detection of Gallbladder Carcinoma
}

This article was published in the following Dove Press journal:

Cancer Management and Research

\author{
Weihao Kong $\mathbb{D}^{1, *}$ \\ Li Zhang ${ }^{2, *}$ \\ Ran $\mathrm{An}^{1, *}$ \\ Mingwei Yang ${ }^{2}$ \\ Hao Wang ${ }^{2}$
}

'Department of Emergency Surgery, Department of Emergency Medicine, The First Affiliated Hospital of Anhui Medical University, Hefei, People's Republic of China; ${ }^{2}$ Department of Radiation Oncology, The First Affiliated Hospital of Anhui Medical University, Hefei, People's Republic of China

*These authors contributed equally to this work
Correspondence: Hao Wang

Department of Radiation Oncology, The First Affiliated Hospital of Anhui Medical University, 218 Jixi Avenue, Hefei, 230032, People's Republic of China Email wanghaoayfy@163.com
Background: Previous studies have shown that D-dimer plays an essential role in the occurrence and development of various tumors, and its diagnostic value in gallbladder carcinoma (GBC) is unknown. Therefore, the purpose of our study was to explore the diagnostic value of $\mathrm{D}$-dimer in distinguishing between gallbladder carcinoma and benign controls.

Methods: We retrospectively included age and gender-matched patients with gallbladder carcinoma and benign gallbladder lesions, and analyzed the diagnostic value of inflammatory markers, D-dimers, and tumor biomarkers by receiver operating characteristic curves (ROC).

Results: The area under the ROC curve of white blood cells (WBC), neutrophil to lymphocyte ratio (NLR), platelet to lymphocyte ratio (PLR), D-dimer, alpha-fetoprotein (AFP), carcinoembryonic antigen (CEA), and carbohydrate antigen 19-9 (CA19-9) were $0.600,0.760,0.729,0.849,0.502,0.699$, and 0.802 , respectively. The combined diagnostic value of D-dimer and CA19-9 was 0.920, which was superior to other joint indicators.

Conclusion: Serum D-dimer may be considered as a potential biomarker for detection of GBC. Moreover, the combined diagnosis of D-dimer and CA19-9 has excellent diagnostic value in gallbladder carcinoma.

Keywords: D-dimer, CA19-9, diagnosis, gallbladder carcinoma

\section{Introduction}

Gallbladder carcinoma (GBC) is the fifth most common malignant tumors in the digestive system and the most common type of biliary malignancy. ${ }^{1}$ According to epidemiological statistics, the incidence of gallbladder carcinoma in China was $52.8 / 100000$ in 2015 , and the mortality rate was $40.7 / 100000$. Its 5-year survival rate is only $5 \%$, and the average overall survival time is six months. ${ }^{2}$ Due to the high invasiveness of gallbladder carcinoma and the lack of typical specific symptoms in the early stage, most of the first diagnosed patients are already in the advanced stage, and the best time for surgical resection has been missed. $^{3,4}$

At present, the diagnosis of gallbladder carcinoma mainly relies on laboratory and imaging examinations for comprehensive evaluation. Ultrasound is one of the conventional screening methods for gallbladder carcinoma, but its ability to detect early lesions is weak. Magnetic resonance imaging (MRI) and computed tomography (CT) are useful for distinguishing gallbladder carcinoma from other benign diseases, but their long appointment period and radioactivity may 
partially limit their widespread use. Besides, tumor markers such as carbohydrate antigen 19-9 (CA19-9) and carcinoembryonic antigen (CEA) are also used for screening for gallbladder carcinoma, but their diagnostic efficacy is not often satisfactory. Therefore, finding a high-efficient, economical, and sensitive biomarker for early diagnosis of gallbladder carcinoma has important clinical significance.

D-dimer is a specific degradation product produced by the hydrolysis of cross-linked fibrin monomer. ${ }^{5,6}$ D-dimer was initially found to be a vital component in the process of hemostasis. ${ }^{7}$ Recent studies have shown that D-dimer is significantly associated with the staging and prognosis of a variety of cancers, its diagnostic value in distinguishing between gallbladder carcinoma and benign controls has not been explored so far. Therefore, in this study, we included age and gender-matched patients with gallbladder carcinoma and benign lesions to investigate the diagnostic efficacy of D-dimer in the detection of GBC and compared it with other inflammatory markers and tumor biomarkers.

\section{Patients and Methods}

\section{Patients}

We retrospectively collected the hematological parameters of 61 patients with pathologically diagnosed gallbladder carcinoma who underwent surgery between January 2016 and May 2019 in the First Affiliated Hospital of Anhui Medical University. The inclusion criteria for this study were: (1) the patient underwent surgical resection or needle biopsy and histopathology confirmed gallbladder carcinoma; (2) the patient received the detection of tumor biomarker included CA19-9; (3) the patient did not receive radiotherapy or chemotherapy before surgery. Exclusion criteria were: blood system diseases, infectious diseases, autoimmune diseases, accompanying other malignant tumors, and the use of anticoagulants. Besides, gender and age-matched benign gallbladder lesions (including gallbladder polyps and cholecystitis) were used as controls for GBC. The research protocol was authorized by the ethics committee review agency of the First Affiliated Hospital of Anhui Medical University (ethics number: P2020-16-17).

\section{Laboratory Testing and Clinical Data}

Laboratory indicators and clinicopathological features were obtained from hospitalized medical records. We extracted laboratory indicators within one week prior to surgical resection. Laboratory indicators include white blood cell count, neutrophil count, lymphocyte count, platelet count, D-dimer, AFP, CEA, and CA19-9. Clinicopathological features including age, sex, degree of differentiation, depth of tumor invasion, number of lymph node metastases, distant metastasis, and tumor stage. The neutrophil count divided by the lymphocyte count is defined as NLR, and the platelet count divided by the lymphocyte count is defined as PLR. The tumor stage of GBC was determined according to the American Cancer TNM Staging System Joint Committee (8th Edition) classification system. ${ }^{8,9}$ $\mathrm{T}$ refers to the primary tumor stage, which is divided into Tis, T1a, T1b, T2a, T2b, T3, and T4 on account of the depth of tumor invasion. $\mathrm{N}$ refers to regional lymph node metastasis, which is divided into N0, N1, and N2. N0 refers to the absence of local lymph node metastasis, N1 refers to the occurrence of 1-3 lymph node metastasis, and $\mathrm{N} 2$ refers to $\geq 4$ lymph node metastasis. $\mathrm{M}$ means there is distant metastasis, M0 means there is no distant metastasis, and M1 means there is distant metastasis.

\section{Statistical Analysis}

SPSS 24.0 and GraphPad prism7.0 were utilized for statistical analysis. The categorical variables were shown by frequency, and the continuous variables are expressed using the median (interquartile range) if they do not satisfy the normal distribution, and the mean \pm standard deviation is used for normal distribution. When comparing the two groups, chi-square analysis is used if the variable is a categorical variable; the Mann-Whitney $U$-test is used if the variable is a continuous variable and does not satisfy the normal distribution, and a $t$-test is used for continuous variable that satisfying the normal distribution. The MedCalc software was used to calculate the optimal cutoff value, sensitivity (SEN), specificity (SPE), positive likelihood ratio (PLR), negative likelihood ratio (NLR), and area under the ROC curve for laboratory indicators. When the P-value is less than 0.05 , it is considered to be statistically significant. 
Table I Clinicopathological Features and Laboratory Indicators of the Participants

\begin{tabular}{|c|c|c|c|}
\hline Variables & GBC & Benign Control & P-value \\
\hline Age (year) & $n=61$ & $n=61$ & 0.138 \\
\hline$<60$ & $20(32.8)$ & $28(46.0)$ & \\
\hline$\geq 60$ & $4 \mid(67.2)$ & $33(54.0)$ & \\
\hline Gender & & & 0.855 \\
\hline Male & $27(44.3)$ & $26(42.6)$ & \\
\hline Female & $34(55.7)$ & $35(57.4)$ & \\
\hline Differentiation & & & - \\
\hline Well & $4(6.6)$ & - & \\
\hline Moderate & $41(67.2)$ & - & \\
\hline Poor & $12(19.6)$ & - & \\
\hline NA & $4(6.6)$ & - & \\
\hline $\mathrm{T}$ stage & & & - \\
\hline Tis-T2 & $24(39.3)$ & - & \\
\hline T3 & $27(44.3)$ & - & \\
\hline T4 & $10(16.4)$ & - & \\
\hline $\mathrm{N}$ stage & & & - \\
\hline No & $36(59.0)$ & - & \\
\hline NI & $16(26.2)$ & - & \\
\hline N2 & $9(14.8)$ & - & \\
\hline Metastasis & & & - \\
\hline Mo & $51(83.6)$ & - & \\
\hline MI & $10(16.4)$ & - & \\
\hline \multicolumn{4}{|l|}{ Stage } \\
\hline $0-11$ & $18(29.5)$ & - & - \\
\hline III & $27(44.3)$ & - & \\
\hline IV & $16(26.2)$ & - & \\
\hline WBC (10^9/L) & $5.85(5.11-7.83)$ & $5.74(4.63-6.35)$ & 0.056 \\
\hline NLR & $2.58(1.86-3.60)$ & $1.74(1.25-2.19)$ & $<0.001$ \\
\hline PLR & $164.25 \pm 72.83$ & $\mid 12.21 \pm 40.47$ & $<0.001$ \\
\hline D-dimer(ug/mL) & $0.70(0.46-1.37)$ & $0.31(0.24-0.44)$ & $<0.001$ \\
\hline AFP $(\mathrm{ng} / \mathrm{mL})$ & $3.10(2.10-4.00)$ & $2.90(2.20-4.28)$ & 0.971 \\
\hline CEA $(\mathrm{ng} / \mathrm{mL})$ & $3.30(1.90-6.75)$ & $2.13(1.45-3.20)$ & $<0.001$ \\
\hline CAI9-9 (U/mL) & $55.4 \mid(\mid 3.28-843.9)$ & $9.41(5.06-17.26)$ & $<0.001$ \\
\hline
\end{tabular}

Note: Bold font represents the P-value less than 0.05 .

Abbreviations: NA, not available; GBC, gallbladder carcinoma; WBC, white blood cell; NLR, neutrophil to lymphocyte ratio; PLR, platelet to lymphocyte ratio; APF, alpha-fetoprotein; CEA, carcinoembryonic antigen.

\section{Results}

\section{Clinical Characteristics of the Study Population}

In our retrospective study, 61 patients with gallbladder carcinoma and 61 benign controls (including 41 cholecystitis controls and 20 polyp controls) were enrolled. Table 1 demonstrates the clinicopathological features and laboratory indicators of all included populations. In GBC patients, ten patients had distant metastases. Among the ten patients with GBC metastases, four patients had abdominal wall metastases, and six patients had gastrointestinal, inguinal, and omental metastases. The abdominal wall and groin's metastatic lesions are single, while the metastatic lesions of the gastrointestinal tract and omentum are multiple. According to the AJCC (8th edition) stage system, 17 people belong to stage IV, 26 people belong to stage III, and 18 people belong to stage 0 -II. The results of chi-square analysis showed no significant statistical difference in age and gender between the GBC group and the benign control group. NLR, PLR, D-dimer, CEA, and CA199 were statistically significant between the two groups based on the results of the $t$-test and MannWhitney $U$-test. Furthermore, we found that D-dimer and CA199 are significantly higher in patients with gallbladder carcinoma than in patients with gallbladder polyps and cholecystitis (Figure 1).

\section{Correlation Between Clinicopathological Features and D-Dimer/CA19-9 Levels in GBC}

The correlation between the levels of pre-operative D-dimer and CA19-9 and the clinicopathological features of gallbladder carcinoma are demonstrated in Table 2. Correlation analysis showed that D-dimer was significantly associated with depth of tumor invasion ( $\mathrm{P}=0.017)$, and distant metastasis $(\mathrm{P}=0.014)$; while $\mathrm{CA} 19-9$ was significantly associated with gender $(\mathrm{P}=0.043)$, lymph node metastasis $(\mathrm{P}=0.008)$, and tumor stage $(\mathrm{P}=0.004)$.

\section{Diagnostic Value of Laboratory Indicators for Detecting GBC}

To further explore the efficacy of different laboratory indicators in the diagnosis of gallbladder carcinoma, we performed ROC curve analysis. The results showed that the areas under the ROC curves of WBC, NLR, PLR, D-dimer, AFP, CEA, and CA19-9 were 0.600, 

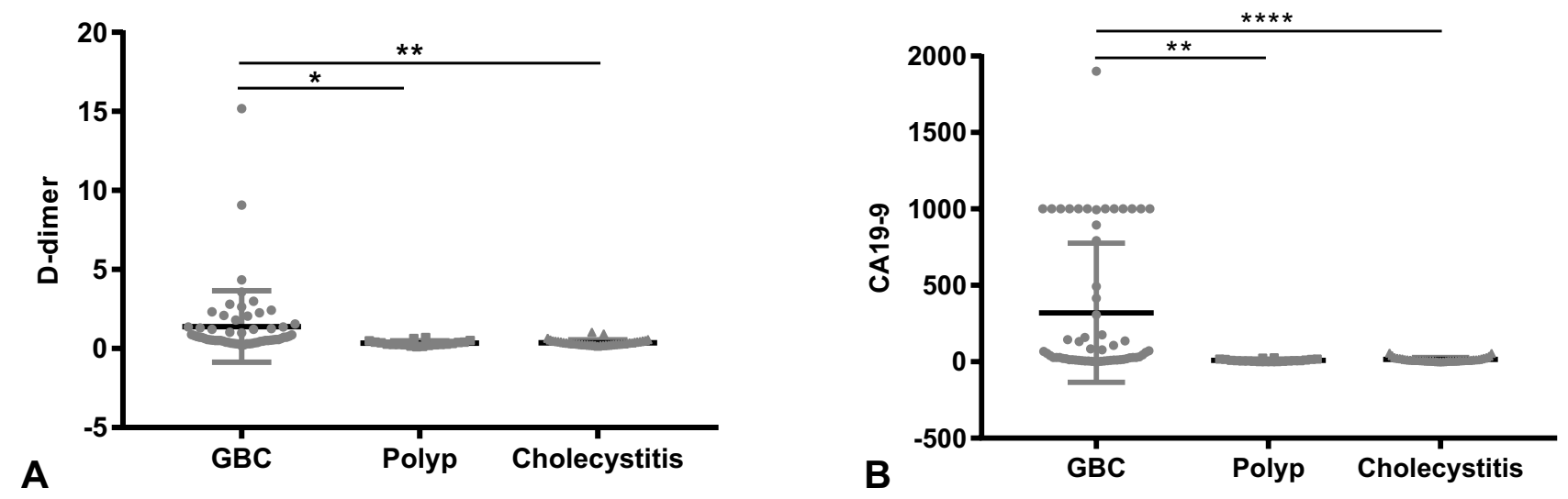

Figure I The serum D-dimer (A) and CAI9-9 (B) in GBC, gallbladder polyp, and cholecystitis controls. Notes: ${ }^{*} \mathrm{P}<0.05, * * \mathrm{P}<0.01, * * * * \mathrm{P}<0.001$.

$0.760,0.729,0.849 .0 .502,0.699$ and 0.802 , respectively, of which D-dimer had the highest diagnostic efficiency in differentiating GBC from benign controls. Among the seven biomarkers, AFP has the lowest sensitivity and specificity, while NLR, PLR, D-dimer, and CA19-9 have relatively high sensitivity and specificity, of which D-dimer has the highest sensitivity, CA19-9 has the highest specificity (Table 3 and Figure 2). Therefore, we combined these indicators using logistic regression, and the results of the combined diagnosis showed that the combination of D-dimer and CA19-9 has higher sensitivity and specificity, which can obviously discriminate GBC and benign gallbladder lesions (Table 4 and Figure 3).

\section{Discussion}

The D-dimer is a fibrinogen degradation product, and the presence of D-dimer indicates the presence of activated thrombus formation and subsequent fibrinolytic activity in the body. Plasma D-dimer has been widely used to diagnose pulmonary embolism, disseminated intravascular coagulation, myocardial infarction and thromboembolism. ${ }^{10-15}$ Recent studies have shown that D-dimers are dysregulated in many different types of cancers, including gastric cancer, musculoskeletal sarcoma, lung cancer, pancreatic cancer, esophageal squamous cell carcinoma, ovarian cancer, nasopharyngeal cancer, liver cancer, lymphoma, spinal giant cell tumor, and breast cancer. ${ }^{6,7,16-26}$ Moreover, several studies have shown that elevated levels of D-dimer are significantly associated with cancer recurrence, metastasis, and worse survival outcome. ${ }^{6,17,24-26}$ However, to date, no one has studied the diagnostic value of $\mathrm{D}$-dimer in gallbladder carcinoma. Therefore, we retrospectively included age and gender matched gallbladder carcinoma patients and benign controls to explore the diagnostic value of D-dimer in the detection of gallbladder carcinoma.

In our study, we found that serum D-dimer and CA19-9 levels were significantly higher in patients with gallbladder carcinoma than patients with gallbladder polyps and cholecystitis, suggesting that D-dimer and CA19-9 are differentially expressed between GBC and benign controls. Then, we analyzed the correlation between D-dimer/CA19-9 and clinicopathological features. The results of the correlation analysis showed that the expression level of D-dimer was significantly correlated with invasion depth $(\mathrm{P}=0.017)$, and distant metastasis $(\mathrm{P}=0.014)$ of $\mathrm{GBC}$. The expression level of CA19-9 was significantly correlated with lymph node invasion $(\mathrm{P}=0.008)$ and TNM stage $(\mathrm{P}=0.004)$ of GBC. Hence, we can speculate that tumor cells may induce the secretion of D-dimers to promote tumor proliferation, local invasion, and metastasis. Similarly, CA19-9 is expressed more highly in patients with gallbladder carcinoma with lymphatic invasion and higher tumor stage, suggesting that CA19-9 secreted by tumor cells may be associated with lymph node invasion and progression of gallbladder carcinoma. Finally, we performed a receiver operating characteristic curve analysis, and the results showed that D-dimer have a good diagnostic efficiency than CA19-9 in 
Table 2 Association Between Clinicopathological Features and D-Dimer or CAI9-9 in GBC

\begin{tabular}{|c|c|c|c|c|}
\hline Variables & D-Dimer & P-value & CAI9-9 & P-value \\
\hline Age (year) & & 0.080 & & 0.805 \\
\hline$<60$ & $0.58(0.31-1.26)$ & & $44.85(12.29-493.20)$ & \\
\hline$\geq 60$ & $0.72(0.52-1.93)$ & & $61.15(13.28-945.20)$ & \\
\hline Gender & & 0.172 & & 0.043 \\
\hline Male & $0.58(0.46-1.26)$ & & $27.23($ (II.08-II3.30) & \\
\hline Female & $0.85(0.50-1.62)$ & & $140.85(25.67-1000.00)$ & \\
\hline Pathology type & & 0.207 & & 0.274 \\
\hline Adenocarcinoma & $0.61(0.45-1.37)$ & & 69.70 (I4.97-869.55) & \\
\hline Others & $0.89(0.7 \mathrm{I}-\mathrm{I} .48)$ & & 24.24 (2.77-765.29) & \\
\hline Differentiation & & 0.290 & & 0.323 \\
\hline Well & $0.48(0.34-11.5)$ & & $|2.3|(8.65-34.62)$ & \\
\hline Moderate & $0.58(0.43-1.26)$ & & $67.92(20.52-843.9)$ & \\
\hline Poor & $0.97(0.70-2.04)$ & & $78.21(4.24-1000.00)$ & \\
\hline NA & $1.61(0.52-2.90)$ & & $503.98(5.17-1000.00)$ & \\
\hline T stage & & 0.017 & & 0.065 \\
\hline Tis-T2 & $0.55(0.34-0.85)$ & & 27.81 (9.68-140.73) & \\
\hline T3 & $0.72(0.5 \mathrm{I}-1.55)$ & & $107.30(21.92-1000.00)$ & \\
\hline $\mathrm{T} 4$ & $1.59(0.67-2.68)$ & & $539.11(16.35-1000.00)$ & \\
\hline$N$ stage & & 0.091 & & 0.008 \\
\hline No & $0.61(0.4 I-1.29)$ & & 27.98 (II.46-8I.39) & \\
\hline NI & $0.67(0.41-0.88)$ & & $642.90(113.30-1000.00)$ & \\
\hline N2 & $2.10(0.55-2.89)$ & & $78.21(8.00-1000.00)$ & \\
\hline Metastasis & & 0.014 & & 0.883 \\
\hline Mo & $0.58(0.40-1.22)$ & & $55.4 \mid(\mid 3.62-493.20)$ & \\
\hline MI & $2.08(0.65-2.85)$ & & $52.57(8.03-1000)$ & \\
\hline Stage & & 0.059 & & 0.004 \\
\hline $0-I I$ & $0.56(0.32-1.10)$ & & I6.31 (9.09-47.49) & \\
\hline III & $0.72(0.5 \mathrm{I}-\mathrm{I} .22)$ & & $107.3(21.92-895.20)$ & \\
\hline IV & $1.59(0.54-2.55)$ & & $746.60(21.06-1000.00)$ & \\
\hline
\end{tabular}

Note: Bold font represents the P-value less than 0.05 .

Abbreviations: NA, not available; GBC, gallbladder carcinoma. 
Table 3 Diagnosis Value of Single Laboratory Indicators for GBC Detection

\begin{tabular}{|l|c|c|c|l|l|l|}
\hline Variables & Cut-Off value & Sensitivity & Specificity & LR+ & LR- & AUC \\
\hline WBC & 6.46 & 40.98 & 80.33 & 2.08 & 0.73 & $0.600(0.508-0.688)$ \\
\hline NLR & 2.06 & 70.49 & 73.77 & 2.69 & 0.40 & $0.760(0.675-0.833)$ \\
\hline PLR & 140.40 & 59.02 & 83.61 & 3.60 & 0.49 & $0.729(0.641-0.805)$ \\
\hline D-dimer & 0.49 & 73.77 & 85.25 & 5.00 & 0.31 & $0.849(0.773-0.907)$ \\
\hline AFP & 2.91 & 49.18 & 44.26 & 0.79 & 1.26 & $0.502(0.410-0.594)$ \\
\hline CEA & 3.60 & 65.57 & 81.97 & 2.73 & 0.62 & $0.699(0.609-0.778)$ \\
\hline CA19-9 & 26.60 & 90.16 & 6.67 & 0.38 & $0.802(0.721-0.869)$ \\
\hline
\end{tabular}

Abbreviations: WBC, white blood cell; NLR, neutrophil to lymphocyte ratio; PLR, platelet to lymphocyte ratio; APF, alpha-fetoprotein; CEA, carcinoembryonic antigen; LR + , positive likelihood ratio; LR-, negative likelihood ratio; AUC, area under the receiver operating characteristic curve.

distinguishing gallbladder carcinoma from benign control disease, and the combined diagnostic efficiency is higher in GBC detection. Both of them may have important clinical significance in the diagnosis of

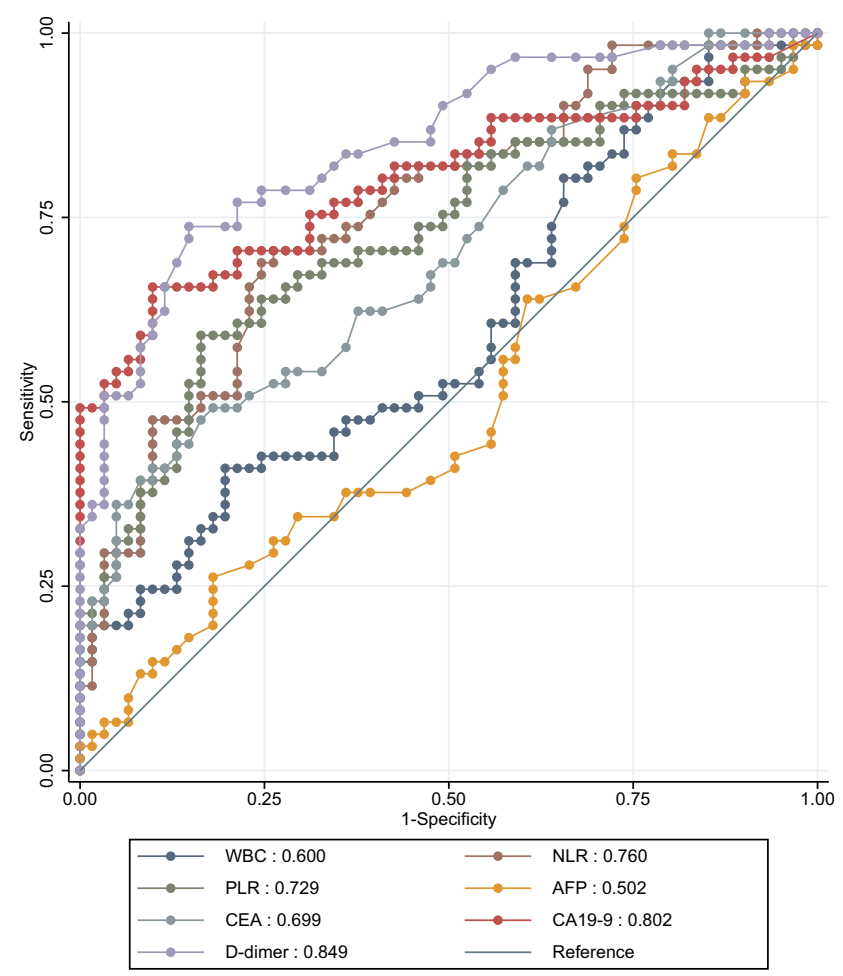

Figure 2 Receiver operating characteristic curve (ROC) of single laboratory indicators for GBC detection.

Abbreviations: WBC, white blood cell; NLR, neutrophil to lymphocyte ratio; PLR, platelet to lymphocyte ratio; APF, alpha-fetoprotein; CEA, carcinoembryonic antigen; CA19-9, carbohydrate antigen 19-9. gallbladder carcinoma. Our results are consistent with Chen's research, and he found that D-dimer were significantly higher in patients with ovarian cancer than in patients with endometriosis and had good diagnostic efficacy in differentiating ovarian cancer from endometriosis. ${ }^{27}$

The underlying mechanism by which D-dimers play a role in the progression of gallbladder carcinoma may be as follows: D-dimer is a biomarker that suggests activation of hemostasis and fibrinolysis. It is a degradation product of fibrin mediated by plasmin. Tumor cells can activate the coagulation system by producing procoagulant factors, resulting in a hypercoagulable state of the body. Due to the enhanced procoagulant state, the fibrinolytic system is then activated, so the expression of fibrin degradation products (including D-dimer) is significantly increased in the body, so the increase of D-dimer can be used as an indicator of a hypercoagulable state. ${ }^{5,6}$ Previous studies have shown that hypercoagulable state can promote tumor proliferation, invasion, migration, and angiogenesis. ${ }^{28}$ Therefore, D-dimer may promote the progression of the tumor by promoting the hypercoagulable state of the body.

At the same time, there are shortcomings in our research. First, this is a retrospective study, and even if we match age and gender, selective bias is inevitable Second, the sample size we included in the study was small, and subsequent large-scale, multi-center research data was needed to verify the reliability of our results. 
Table 4 Diagnosis Value of Combined Laboratory Indicators for GBC Detection

\begin{tabular}{|l|l|l|l|l|l|}
\hline Variables & Sensitivity & Specificity & LR+ & LR- & AUC \\
\hline PLR+D-dimer & 73.77 & 83.61 & 4.50 & 0.31 & $0.858(0.783-0.915)$ \\
\hline NLR+D-dimer & 68.85 & 90.16 & 7.00 & 0.35 & $0.862(0.788-0.918)$ \\
\hline PLR+CAI9-9 & 77.05 & 90.16 & 7.83 & 0.25 & $0.831(0.753-0.893)$ \\
\hline NLR+CA19-9 & 72.13 & 83.61 & 4.40 & 0.33 & $0.854(0.778-0.911)$ \\
\hline D-dimer+CA19-9 & 83.61 & 90.16 & 8.33 & 0.20 & $0.920(0.857-0.962)$ \\
\hline
\end{tabular}

Abbreviations: NLR, neutrophil to lymphocyte ratio; PLR, platelet to lymphocyte ratio; CAI9-9, carbohydrate antigen 19-9; LR+, positive likelihood ratio; LR-, negative likelihood ratio; AUC, area under the receiver operating characteristic curve.

Third, we did not further explore the potential mechanism of D-dimer in the development of gallbladder carcinoma.

In summary, D-dimer and CA19-9 are useful biomarkers for the diagnosis of gallbladder carcinoma, and the combined diagnostic value of the two biomarkers is relatively high, which guides the early detection of gallbladder carcinoma. Considering the limited sample size, more large-scale, multicenter studies are needed for verification.

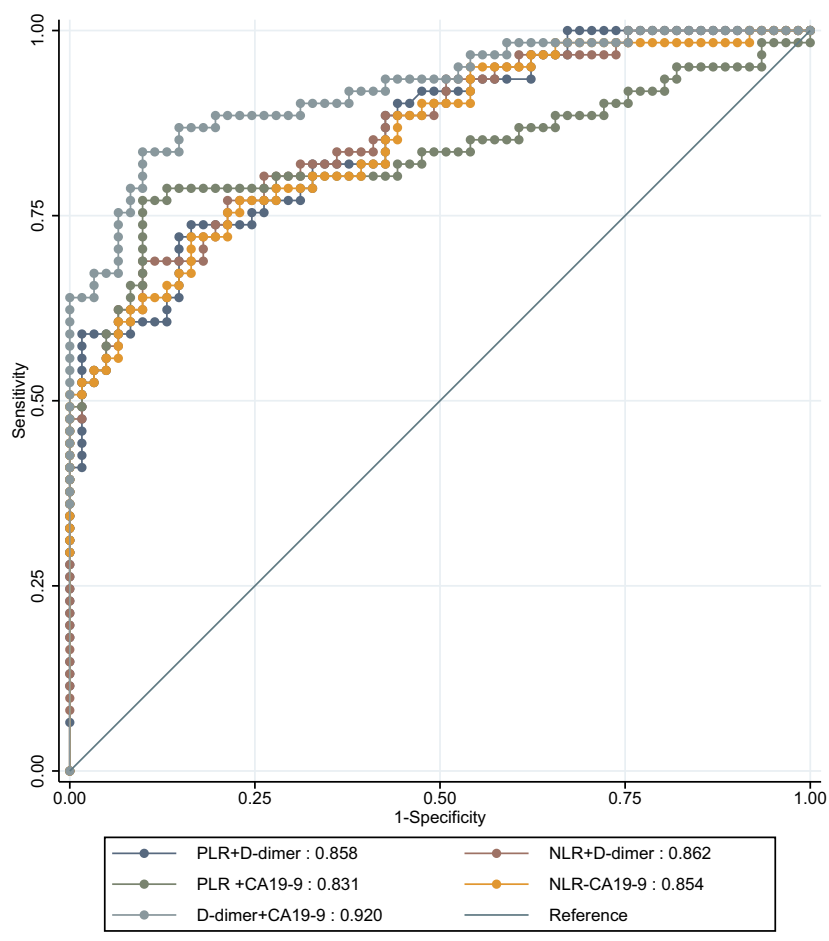

Figure 3 Receiver operating characteristic curve (ROC) of combined laboratory indicators for GBC detection.

Abbreviations: NLR, neutrophil to lymphocyte ratio; PLR, platelet to lymphocyte ratio; CA19-9, carbohydrate antigen 19-9.

\section{Ethics}

The research protocol was authorized by the ethics committee review agency of the First Affiliated Hospital of Anhui Medical University (ethics number: P2020-16-17). Our research complies with the "Declaration of Helsinki." At the same time, we have concealed the patient's personal information and kept the patient's personal information confidential. Therefore, our experiment will not affect the patient's general condition, so the patient is informed consent is exempt.

\section{Acknowledgments}

This work was supported by the 2015 Clinical Science Research Project of Anhui Medical University (Project Number: 2015xkj089) and China International Medical Exchange Fund (Project Number: Z-2014-06-17357).

\section{Disclosure}

The authors report no conflicts of interest in this work.

\section{References}

1. Shukla SK, Singh G, Shahi KS, Bhuvan P, Pant P. Staging, treatment, and future approaches of gallbladder carcinoma. $J$ Gastrointest Cancer. 2018;49(1):9-15. doi:10.1007/s12029-017-0036-5

2. Chen W, Zheng R, Baade PD, et al. Cancer statistics in China, 2015. CA Cancer J Clin. 2016;66(2):115-132. doi:10.3322/caac.21338

3. Goetze TO. Gallbladder carcinoma: prognostic factors and therapeutic options. World J Gastroenterol. 2015;21(43):12211-12217. doi:10.3748/wjg.v21.i43.12211

4. Kakaei F, Beheshtirouy S, Nejatollahi SM, Zarrintan S, Mafi MR. Surgical treatment of gallbladder carcinoma: a critical review. Updates Surg. 2015;67(4):339-351. doi:10.1007/s13304-015-0328-x

5. Rong G, Fan W, Shen J. High pretreatment plasma D-dimer levels predict poor prognosis in gastrointestinal cancers: a meta-analysis. Medicine. 2019;98(29):e16520. doi:10.1097/MD.0000000000016520

6. Zhang Q, Zhang Y, Chen D, et al. Preoperative D-dimer level is an independent prognostic factor for non-small cell lung cancer after surgical resection: a systematic review and meta-analysis. PLoS One. 2019;7(16):366. 
7. Lu Y, Zhang L. The association of D-dimer with clinicopathological features of breast cancer and its usefulness in differential diagnosis: a systematic review and meta-analysis. PLoS One. 2019;14(9):e0221374.

8. Lafaro K, Blakely AM, Melstrom LG, et al. Prognostic impact of tumor location in resected gallbladder cancer: a national cohort analysis. J Surg Oncol. 2020;122:1084-1093. doi:10.1002/jso.26107

9. Lee SE, Choi YS, Kim YH, et al. Prognostic significance of tumor location in T2 gallbladder cancer: a Korea Tumor Registry System Biliary Pancreas (KOTUS-BP) database analysis. J Clin Med. 2020;9 (10):3268. doi: $10.3390 /$ jcm9103268

10. Kim SJ, Kim MH, Lee KM, et al. Troponin I and D-dimer for discriminating acute pulmonary thromboembolism from myocardial infarction. Cardiology. 2017;136(4):222-227. doi:10.1159/000449404

11. Kim SY, Kim JE, Kim HK, Kim I, Yoon SS, Park S. Higher prognostic value of soluble fibrin complexes than D-dimer and fibrin degradation product for disseminated intravascular coagulation in patients with liver cirrhosis. Blood Coagul Fibrinol. 2013;24 (2):150-156. doi:10.1097/MBC.0b013e32835aef6b

12. Li WJ, Sha M, Ma W, Zhang ZP, Wu YJ, Shi DM. Efficacy evaluation of D-dimer and modified criteria in overt and nonovert disseminated intravascular coagulation diagnosis. Int $J$ Lab Hematol. 2016;38(2):151-159. doi:10.1111/ijlh.12467

13. Ohara T, Farhoudi M, Bang OY, Koga M, Demchuk AM. The emerging value of serum D-dimer measurement in the work-up and management of ischemic stroke. Int J Stroke. 2019;1747493019876538.

14. Reihani H, Sepehri Shamloo A, Keshmiri A. Diagnostic value of D-dimer in acute myocardial infarction among patients with suspected acute coronary syndrome. Cardiol Res. 2018;9(1):17-21. doi:10.14740/cr620w

15. Satilmisoglu MH, Ozyilmaz SO, Gul M, et al. Predictive values of D-dimer assay, GRACE scores and TIMI scores for adverse outcome in patients with non-ST-segment elevation myocardial infarction. Ther Clin Risk Manag. 2017;13:393-400. doi:10.2147/TCRM. S124794

16. Chen X, Chang Z, Liu Z. D-dimer increase: an unfavorable factor for patients with primary liver cancer treated with TACE. Eur Spine J. 2019;83(4):797-802.

17. Fan S, Zhao G, An G. High pretreatment plasma D-dimer levels are associated with shorter overall survival in patients with small cell lung cancer. J Int Med Res. 2019;47(1):215-224. doi:10.1177/ 0300060518799869

18. Feng JF, Yang X, Chen S, Zhao Q, Chen QX. Prognostic value of plasma D-dimer in patients with resectable esophageal squamous cell carcinoma in China. J Cancer. 2016;7(12):1663-1667. doi:10.7150/ jca. 15216
19. Geng YD, Chen YR, Jin J, Wang XD, Zhang S, Li DJ. Prognostic value of D-dimer in patients with diffuse large B-cell lymphoma: a retrospective study. Curr Med Sci. 2019;39(2):222-227. doi:10.1007/s11596-019-2023-5

20. He SS, Wang Y, Wang CT, et al. A combined marker based on plasma D-dimer and serum albumin levels in patients with nasopharyngeal carcinoma is associated with poor survival outcomes in a retrospective cohort study. J Cancer. 2019;10(16):3691-3697. doi:10.7150/jca.32387

21. Li B, Zhang H, Zhou P, et al. Prognostic significance of pretreatment plasma D-dimer levels in patients with spinal chordoma: a retrospective cohort study. Eur Spine J. 2019;28(6):1480-1490. doi:10.1007/s00586-018-05872-4

22. Li H, Zhao S, Jing Z, Li J, Shuanying Y, Zhang N. Combination of D-dimer and carcinoembryonic antigen levels as a predictive and prognostic biomarker in advanced colorectal cancer patients. $J$ Cell Biochem. 2019;120(5):8086-8092. doi:10.1002/jcb.28087

23. Morii T, Mochizuki K, Tajima T, Ichimura S, Satomi K. D-dimer levels as a prognostic factor for determining oncological outcomes in musculoskeletal sarcoma. BMC Musculoskelet Disord. 2011;12:250. doi:10.1186/1471-2474-12-250

24. Stender MT, Larsen AC, Sall M, Thorlacius-Ussing O. D-Dimer predicts prognosis and non-resectability in patients with pancreatic cancer: a prospective cohort study. Blood Coagul Fibrinol. 2016;27 (5):597-601. doi:10.1097/MBC.0000000000000559

25. Xu K, Wan W, Li B, et al. Prognostic significance of preoperative plasma D-dimer level and clinical factors in patients with spinal giant cell tumor: retrospective analysis of 153 patients in a single center. World Neurosurg. 2019;122:e872-e880. doi:10.1016/j. wneu.2018.10.169

26. Yamada Y, Kawaguchi R, Iwai K, et al. Preoperative plasma D-dimer level is a useful prognostic marker in ovarian cancer. $J$ Obstet Gynaecol. 2019;1-5.

27. Chen L, Wang X, Shu J, Xu S, Wu Q, Yu Y. Diagnostic value of serum D-dimer, CA125, and neutrophil-to-lymphocyte ratio in differentiating ovarian cancer and endometriosis. Int $J$ Gynaecol Obstet. 2019;147(2):212-218. doi:10.1002/ijgo.12949

28. Arce M, Pinto MP, Galleguillos M, et al. Coagulation factor xa promotes solid tumor growth, experimental metastasis and endothelial cell activation. Cancers. 2019;11(8):1103. doi:10.3390/ cancers 11081103

\section{Publish your work in this journal}

Cancer Management and Research is an international, peer-reviewed open access journal focusing on cancer research and the optimal use of preventative and integrated treatment interventions to achieve improved outcomes, enhanced survival and quality of life for the cancer patient.
The manuscript management system is completely online and includes a very quick and fair peer-review system, which is all easy to use. Visit http://www.dovepress.com/testimonials.php to read real quotes from published authors. 